\title{
Cylicodiscus gabunensis Harms : une espèce prisée dans le commerce international (synthèse bibliographique)
}

\author{
Romaric Ndonda Makemba ${ }^{(1,2)}$, Félicien Tosso ${ }^{(1,3)}$, Christian Moupela ${ }^{(2)}$, \\ Kasso Daïnou ${ }^{(3)}$, Jean-Louis Doucet ${ }^{(1)}$
}

(1) Université de Liège - Gembloux Agro-Bio Tech. Forest is life. Terra Teaching and Research Centre. Passage des Déportés, 2. BE-5030 Gembloux (Belgique).E-mail : r.n.makemba@gmail.com

(2) Université des Sciences et Techniques de Masuku. Institut National Supérieur d'Agronomie et de Biotechnologies. BP 941. Franceville (Gabon).

(3) Nature ${ }^{+}$asbl s/c Forest is life. TERRA Teaching and Research Centre. Passage des Déportés, 2. BE-5030 Gembloux (Belgique).

Reçu le 12 septembre 2018, accepté le 13 juin 2019, mis en ligne le 14 aout 2019.

Cet article est distribué suivant les termes et les conditions de la licence CC-BY (http://creativecommons.org/licenses/by/4.0/ deed.fr)

Introduction. En raison de la diminution des ressources en bois d'œuvre tropicaux, il convient d'améliorer les connaissances sur les espèces ligneuses en vue de développer des politiques d'exploitation réellement durables. Reconnu pour la grande qualité de son bois, Cylicodiscus gabunensis Harms (Fabaceae-Caesalpinioideae) est une essence à haute valeur socio-économique. Cet article dresse la synthèse bibliographique des connaissances relatives à cette espèce en vue de mettre en avant l'ensemble des aspects méritant des investigations scientifiques approfondies.

Littérature. Commercialisée sous le nom d'okan, $C$. gabunensis est une espèce ligneuse non grégaire vivant dans les forêts denses humides tropicales sempervirentes et semi-décidues. Arbre fétiche pour certains peuples autochtones, $C$.gabunensis est utilisé par les communautés rurales pour de multiples usages. C'est une espèce à phénologie régulière avec une dispersion anémochore des graines. Les populations d'arbres affichent un déficit de régénération en forêt dense humide sempervirente, ce qui compromettrait l'exploitation de l'espèce à long terme. Ce risque est accru par le manque évident d'informations écologiques et sylvicoles permettant une gestion durable.

Conclusions. Cette revue bibliographique résume l'ensemble des informations disponibles sur C. gabunensis principalement en botanique, anatomie du bois, écologie et ethnobotanique. Elle renseigne sur l'état actuel des connaissances au regard des rythmes d'exploitation et de l'état des populations de l'espèce. Des informations complémentaires sont nécessaires pour (i) statuer sur la conservation des populations de l'espèce et (ii) proposer des stratégies de gestion adaptées.

Mots-clés. Cylicodiscus gabunensis, Mimosoideae, botanique, écologie, aménagement durable, structure de population, régénération naturelle, sylviculture.

\section{Cylicodiscus gabunensis Harms: a popular species in international trade. A review}

Introduction. Due to the decline in tropical timber resources, silvicultural and ecological knowledge in these taxa need to be improved for management decision-making. Cylicodiscus gabunensis Harms (Fabaceae-Caesalpinioideae) is a timber tree species with a high socio-economic value. The present paper provides a species-specific literature review and highlights research areas for future investigations.

Literature. Known as okan, C. gabunensis inhabits evergreen and semi-deciduous tropical rain forests. Considered as a sacred tree by indigenous peoples, it is a multipurpose timber tree species. The phenology of this species is regular and its seeds are dispersed by wind. Nowadays, most C. gabunensis populations found in evergreen forests exhibit a clear lack of regeneration, which may threaten its long-term logging. The lack of other relevant silvicultural and ecological information for forest managers may increase that risk of unsustainable exploitation.

Conclusions. This review summarizes the information available on C.gabunensis in the fields of botany, wood anatomy, ecology and ethnobotany. It provides valuable information for forest managers and decision-makers while considering exploitation intensities and tree population characteristics. Highlighting this information is the first step to (i) deciding on a conservation status for the species, and (ii) proposing appropriate management strategies. 
Keywords. Cylicodiscus gabunensis, Mimosoideae, botany, ecology, sustainable management, population structure, natural regeneration, silviculture.

\section{INTRODUCTION}

En Afrique centrale, les facteurs environnementaux et anthropiques ont façonné la composition, la structure et la dynamique des forêts denses humides tropicales (Oslisly et al., 2013 ; Morin-Rivat, 2017). Aujourd'hui, la canopée de ces forêts est souvent dominée par de grands arbres héliophiles (Doucet, 2003; Bourland, 2013) dont certains sont exploités pour leur bois très recherché sur le marché international (Doucet, 2003). Plusieurs études (par exemple Engone Obiang et al., 2011; Bourland, 2013; Biwolé, 2015) ont mis en évidence que la structure démographique des populations de ces essences héliophiles résultait d'un déficit de régénération naturelle.

De la famille des Fabaceae (Leguminosae), sous-famille des Caesalpinioideae (LPWG 2017), Cylicodiscus gabunensis Harms est une de ces espèces héliophiles de grande importance socio-culturelle et économique (Doucet, 2003 ; Meunier et al., 2015 ; Doucet et al., 2016). Elle figure au sixième rang des essences les plus exploitées du bassin du Congo (FRM, 2018). À l'instar des autres essences demandeuses en lumière, elle semble être confrontée à la difficulté de se régénérer naturellement en forêt dense (Swaine \& Hall, 1988 ; Hawthorne, 1995 ; Doucet, 2003 ; Kouadio, 2009), ce qui pourrait compromettre son maintien sur une partie de son aire de distribution. Par ailleurs, les connaissances sur l'écologie et la sylviculture de l'espèce semblent extrêmement limitées (Ayarkwa \& Owusu, 2008). Une meilleure caractérisation des populations de C.gabunensis et de son écologie est donc nécessaire pour assoir des stratégies de gestion durable de ses populations. Cet article dresse une synthèse bibliographique des connaissances actuelles sur cette espèce ligneuse, tout en mettant en avant l'ensemble des aspects méritant des investigations scientifiques approfondies.

Les recherches bibliographiques ont été menées en consultant les bases de données fournies par les moteurs de recherche Google Scholar, Science Direct et Scopus, ainsi que les ressources documentaires disponibles dans la bibliothèque de Gembloux Agro-Bio Tech (Université de Liège). Les termes clés employés en français ainsi que leur équivalent en anglais ont été les suivants: Cylicodiscus gabunensis, Piptadenia gabunensis, Erythrophleum letestui, Erythrophleum gabunense, écologie, aire de distribution, sylviculture, plantation, multiplication, croissance, biomasse, tarif de cubage, germination, phénologie, structure de population, ethnobotanique, aménagement et exploitation forestière.

\section{HISTORIQUE ET TAXONOMIE}

Espèce unique de son genre, C.gabunensis est endémique du continent africain. Elle a été collectée pour la première fois au Gabon par Hermann Soyaux en 1880. En 1894, Paul Hermann Wilhelm Taubert la dénomma Erythrophleum gabunense Taub., mais sans description. Les débats taxonomiques sur les caractéristiques du fruit ont conduit à des changements successifs de dénomination: Cyrtoxiphus staudtii Harms et Cylicodiscus gabunensis Harms en 1897, et Piptadenia gabunensis (Harms) Roberty en 1954. Guy Edouard Roberty ayant finalement établi que les espèces du genre Piptadenia étaient caractérisées par une absence de disque entre les étamines et la base du gynophore, et par des fruits dont la longueur ne dépassait pas $33 \pm 14 \mathrm{~cm}$ (écart-type), décida de confirmer l'espèce dans le genre Cylicodiscus. Du grec kylikis et diskos signifiant "petit disque », le genre Cylicodiscus est donc caractérisé par la présence d'un disque floral (Hédin, 1929; Quattrocchi, 2012). Le nom Cylicodiscus gabunensis Harms a donc été rétabli et reste celui actuellement reconnu.

D'un point de vue phylogénétique, Manzanilla \& Bruneau (2012), puis LPWG (2013) classent C. gabunensis dans la sous-famille des Mimosoideae. $\mathrm{Au}$ sein de celle-ci, le genre monospécifique Cylicodiscus apparait isolé au sein de l'arbre phylogénétique (Luckow et al., 2003 ; Kyalangalilwa et al., 2013), son âge étant estimé à environ 41 millions d'années (Bouchenak-Khelladi et al., 2010). Toutefois, LPWG (2017) classe C.gabunensis dans la sousfamille des Caesalpinoideae, dans laquelle le clade des mimosoides a été intégré sur base de la séquence chloroplastique matK. Ainsi, la sous-famille des Caesalpinoideae comporte dorénavant pas moins de 4400 espèces (LPWG, 2017).

\section{DESCRIPTION GÉNÉRALE}

\subsection{Distribution géographique}

Cylicodiscus gabunensis est une espèce guinéocongolaise confinée quasi exclusivement aux souscentres guinéens supérieur et inférieur (Figure 1) (Droissart et al., 2018). Elle est retrouvée de la Côte d'Ivoire à la République du Congo (CTFT, 1955 ; Ayarkwa \& Owusu, 2008). Des incertitudes subsistent quant à la présence de l'espèce en Sierra Léone (Kryn $\&$ Fobes, 1959). Certaines bases de données suggèrent des occurrences de C.gabunensis plus étendues, 


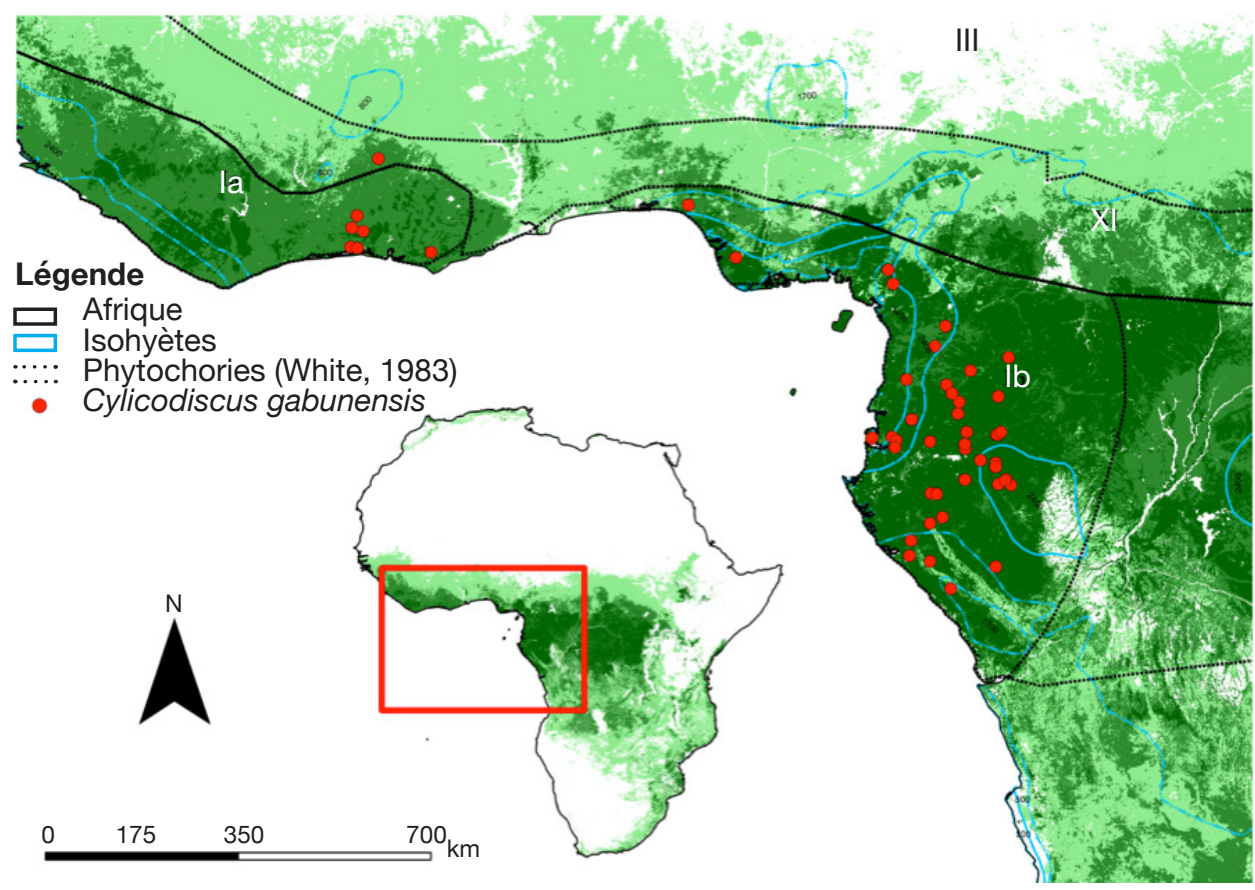

Figure 1. Carte de distribution de Cylicodiscus gabunensis. Les points d'occurrence en rouge proviennent des bases de données du Conservatoire et Jardin botaniques de la Ville de Genève (CJBG, 2018), de South African National Biodiversity Institute (SANBI), du Catalogue of Life Partnership via Global Biodiversity Information Facility (GBIF, 2019) et de la base de données RAINBIO (Dauby et al., 2016). Ia et Ib : région guinéocongolaise, III : région soudanienne, XI : zone de transition (White, 1983). Les localisations douteuses ont été enlevées (voir texte) - Distribution map of Cylicodiscus gabunensis. The occurrence data highlighted in red are provided by the Conservatory of Botanical Gardens of Geneva (CJBG, 2018) and the South African National Biodiversity Institute (SANBI), the Catalogue of Life Partnership via Global Biodiversity Information Facility (GBIF, 2019) and RAINBIO database (Dauby et al., 2016). Ia and Ib: Guinea-Congolese region, III: Sudanese region, XI: transition zone (White, 1983). The questionable locations have been removed (see text).

allant même jusqu'au Kenya (http://www.gbif.org/). Cependant, ces signalements, datant pour la plupart des années cinquante, pourraient résulter de mauvaises identifications, d'autant plus qu'aucun échantillon d'herbier n'est disponible.

\subsection{Description botanique}

La description botanique de $C$. gabunensis est détaillée dans plusieurs flores et autres ressources documentaires spécialisées en botanique : Hédin (1929), CTFT (1955), Aubréville (1959), De Saint Aubin (1963), Letouzey (1982), Vivien \& Faure (1985), Villiers (1989), White \& Abernethy (1996), Luckow \& Grimes (1997), Wilks \& Issembé (2000), Hawthorne \& Gyakari (2006), Ayarkwa \& Owusu (2008) et Meunier et al. (2015).

Cylicodiscus gabunensis est un arbre imposant, de très grande taille (Figure 2), pouvant atteindre $60 \mathrm{~m}$ de haut et $300 \mathrm{~cm}$ de diamètre à hauteur de poitrine $(d h p)$. Le fût de C. gabunensis a parfois des empattements développés à la base. Il est élancé, droit et cylindrique, très écailleux et de teinte brun-noirâtre (Figure 3a). À l'état jeune, il est doté d'excroissances en forme de grosses épines brunes qui disparaissent par la suite (vers $40 \mathrm{~cm}$ de $d h p$, figure $3 \mathbf{b})$. La tranche de C. gabunensis est jaunâtre et très fibreuse, épaisse de 5 à $25 \mathrm{~mm}$ (Figure 3c). Elle a une odeur d'oignon pourri et laisse échapper un exsudat translucide jaunâtre. La cime est hémisphérique et étalée. Les feuilles sont composées bipennées. Elles ont une à deux paires de pennes opposées, chacune comportant de cinq à dix foliolules alternes (Figure 3d). Leurs pétiolules sont glabres, canaliculés et longs de 2 à $3 \mathrm{~mm}$. Le limbe est elliptique, lancéolé, glabre et souvent légèrement asymétrique à la base. Sa longueur varie de 4 à $10 \mathrm{~cm}$ et sa largeur de 2 à $5 \mathrm{~cm}$. Il est moins brillant en dessous que sur la face supérieure. Il est acuminé au sommet. La nervure principale, très visible en dessous, est médiane et légèrement concave sur la face supérieure du limbe. Les nervures secondaires sont au nombre de cinq à dix paires. Elles sont latérales,

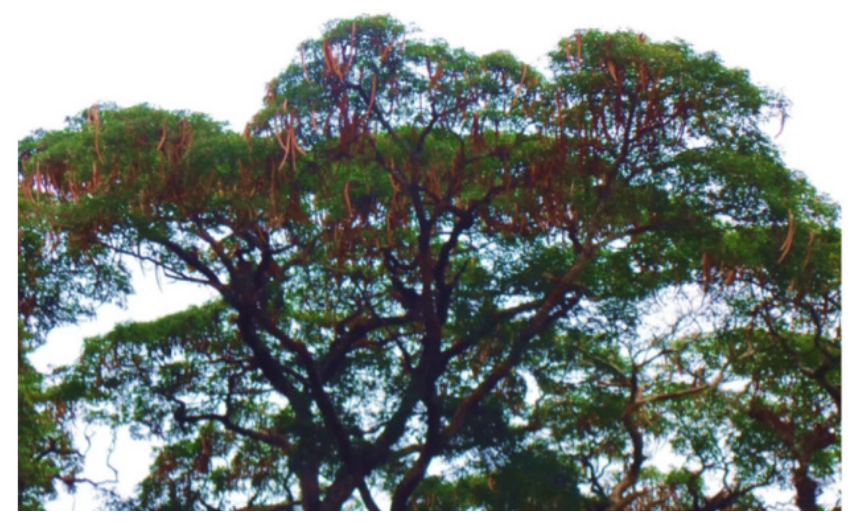

Figure 2. Cime de Cylicodiscus gabunensis portant des fruits en cours de maturation - Crown of a Cylicodiscus gabunensis tree harbouring ripening fruits (C) $\mathrm{R}$. Ndonda Makemba. 

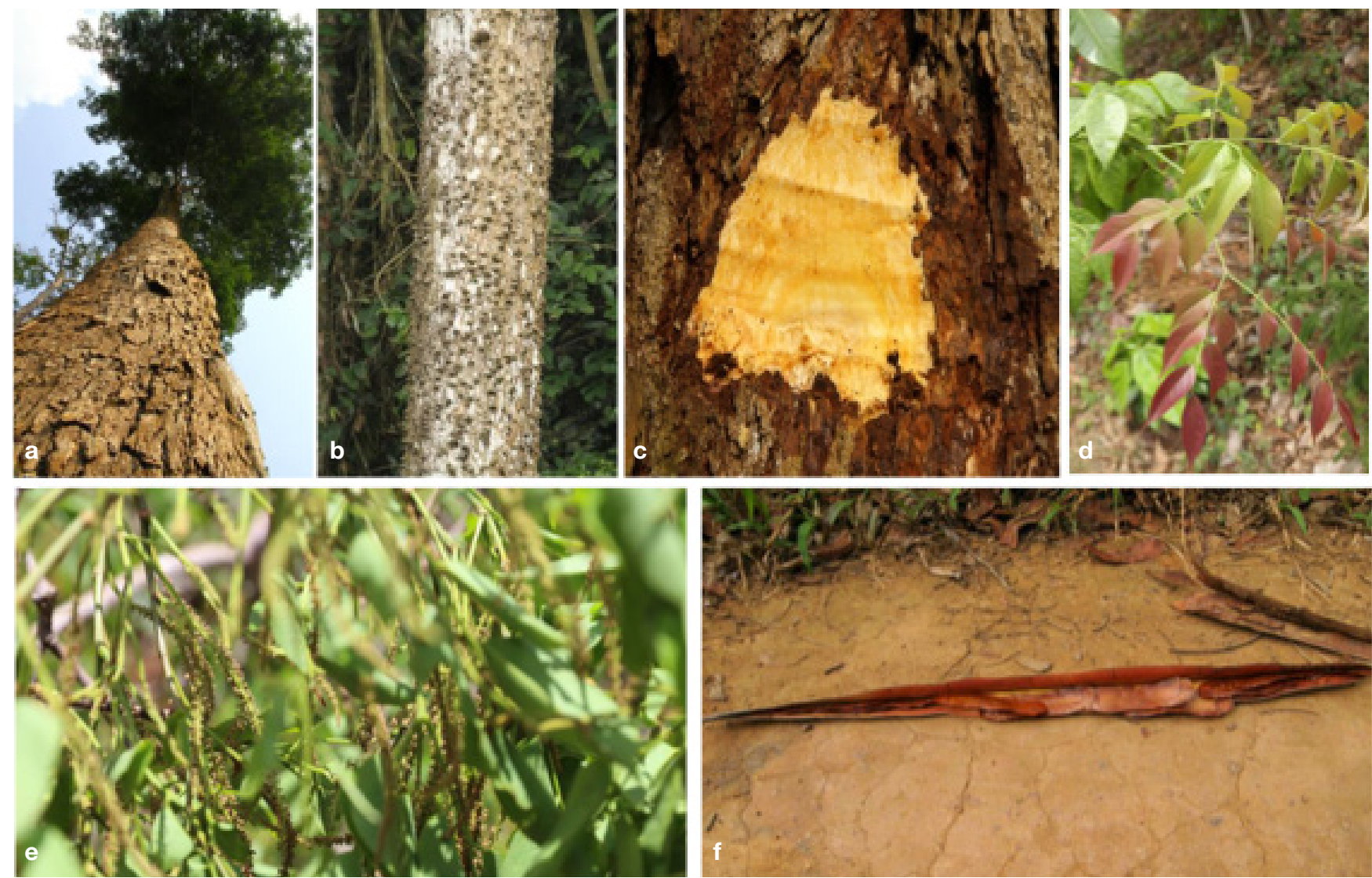

Figure 3. Cylicodiscus gabunensis (C) J.-L. Doucet.

$\mathbf{a}$ : fût d'un individu adulte - trunk of an adult $; \mathbf{b}$ : tronc d'un jeune individu - stem of a sapling ; $\mathbf{c}$ : tranche - slice ; d : feuilles d'un jeune individu - leaves of sapling; $\mathbf{e}$ : rameau en fleurs - flowering twig; $\mathbf{f}$ : fruit et graines matures - fruit and mature seeds.

ascendantes et anastomosées en faible arc à 1,5-3 mm du bord. L'inflorescence est en épis paniculés groupés au sommet des rameaux (Figure 3e). Leurs rachis sont pubescents. Les fleurs hermaphrodites et actinomorphes (à symétrie radiale) sont de couleur blanc jaunâtre. Le calice légèrement denté dispose de cinq sépales soudés entre eux. Ils sont pubescents à l'extérieur et glabres sur la face intérieure. Les cinq pétales glabres sur les deux faces sont oblongs, linéaires de $3 \mathrm{~mm}$ de long et de $0,5 \mathrm{~mm}$ de large. Ils sont libres entre eux et aigus au sommet. Les étamines au nombre de 10 sont libres entre elles. Elles sont longues d'environ $5 \mathrm{~mm}$. Elles disposent d'anthères à deux loges d'environ $400 \mu \mathrm{m}$ de long et terminées au sommet par une pointe glanduleuse caduque et longue de $100 \mu \mathrm{m}$. Le disque intrastaminal remarquable est cylindrique, blanc sur le vivant et long de $1 \mathrm{~mm}$ de haut. L'ovaire pubescent, ellipsoïde est long de $1,5 \mathrm{~mm}$ et surmonté d'un style mince et glabre de $2 \mathrm{~mm}$ de long. Le fruit est une longue gousse coriace de 60 à $100 \mathrm{~cm}$ de long et de 4,5 à $5 \mathrm{~cm}$ de large. Il est étroit, linéaire, aplati avec des nervures réticulées et peu obtus au sommet (Figure 3f). Les nombreuses graines brunes, oblongues, plates peuvent atteindre $7,5 \mathrm{~cm}$ de long. Elles sont attachées à une extrémité et entourées d'une grande aile papyracée (Figure 3f).

\section{4. ÉCOLOGIE ET DYNAMIQUE DES POPULATIONS DE C. GABUNENSIS}

Cylicodiscus gabunensis est une espèce rencontrée dans les climats tropicaux de type humide et de mousson. Elle est limitée aux forêts denses humides sempervirentes et semi-décidues (Ayarkwa \& Owusu, 2008 ; Meunier et al., 2015). Disséminée dans les forêts matures, cette espèce est parfois abondante dans les vieilles forêts secondaires où elle affectionne des sols bien drainés, généralement entre 50 et $630 \mathrm{~m}$ d'altitude (De Saint Aubin, 1963 ; Sosef, 2006 ; Ayarkwa \& Owusu, 2008 ; Herbier National du Gabon, 2019).

\subsection{Tempérament, densités et structures de population}

Tempérament. Selon Doucet (2003), Kouadio (2009) et Meunier et al. (2015), C. gabunensis serait une 
espèce héliophile (qualifiée de pionnière longévive) en Afrique centrale. Toutefois, selon d'autres auteurs, travaillant davantage en Afrique de l'Ouest, l'espèce tolérerait davantage l'ombrage (Hawthorne, 1995; Sheil et al., 2006). Dans les forêts exploitées du Ghana, les plantules de C.gabunensis ont été observées aussi bien en zone non perturbée que dans les chablis et sur les pistes de débardage (Duah-Gyamfi et al., 2014a). Toutefois, bien que figurant parmi les essences commerciales les plus abondantes de la canopée, les densités de semis demeurent très faibles (Duah-Gyamfi et al., 2015), de même que les taux de recrutement (Duah-Gyamfi et al., 2014b). Malheureusement, aucune étude visant à quantifier les besoins en lumière, telle celle conduite par Agyeman et al. (1999), n'a été réalisée.

Selon Vleminckx (2015), des tiges de C. gabunensis $(d h p>30 \mathrm{~cm})$ sont observées là où des charbons de bois sont présents dans le sol, ce qui semble indiquer que l'essence se serait implantée dans des zones ayant subi le passage du feu, probablement d'origine anthropique étant donné que les feux naturels sont extrêmement rares en forêt dense humide tropicale. Selon Biwolé et al. (2015) et Morin-Rivat et al. (2017), l'abondance des espèces héliophiles en forêt dense africaine et l'histoire de l'agriculture itinérante sur brulis seraient étroitement liées. Les espèces héliophiles dominant la canopée de nombreuses forêts africaines se seraient régénérées majoritairement vers la fin du $19^{\mathrm{e}}$ siècle, au moment où les agriculteurs abandonnaient leurs champs sous la contrainte des colons. De Saint Aubin (1963) mentionne d'ailleurs que C.gabunensis est souvent rencontré dans des plantations abandonnées et dans des vieilles forêts secondaires. Les espèces étudiées par Morin-Rivat et al. (2017) ayant des structures de populations comparables à celle de C. gabunensis (voir § ci-dessous), il est donc probable que l'espèce soit héliophile, du moins à un certain stade de développement. Toutefois, des études anthracologiques et archéologiques complémentaires s'avèrent nécessaires pour statuer définitivement.

Densités et structures de population. Les densités de population de C.gabunensis varient selon le type de forêt. Les densités $(d h p \geq 20 \mathrm{~cm})$ observées en forêt dense humide semi-décidue varient de 0,51 à 0,98 tige par hectare. Elles sont globalement plus faibles en forêt sempervirente où elles sont comprises entre 0,04 et 0,50 tige par hectare. Le tableau 1 synthétise les données issues d'inventaires d'aménagements de concessions forestières couvrant 1,8 million d'hectares. De tels inventaires sont particulièrement utiles, pour étudier la répartition spatiale des taxons et sont particulièrement robustes pour les analyses au niveau des genres monospécifiques (Réjou-Méchain et al., 2011).

Les structures des populations de C.gabunensis semblent différer en fonction du type de forêt, avec une allure en cloche caractérisée par un déficit de pieds de faible diamètre en forêt dense humide sempervirente, suggérant une meilleure régénération en forêt semidécidue (exponentielle décroissante) (Figure 4). L'abondance des pieds de gros diamètres indique que les populations de C.gabunensis sont probablement vieillissantes en forêt sempervirente, suggérant que les conditions optimales de régénération des $C$. gabunensis n'y sont plus rencontrées. Plusieurs hypothèses pourraient expliquer les différences observées entre

Tableau 1. Densités des populations de Cylicodiscus gabunensis en forêt tropicale ( $\mathrm{dhp} \geq 20 \mathrm{~cm})-$ Population densities of Cylicodiscus gabunensis in tropical forests $(d b h \geq 20 \mathrm{~cm})$.

\begin{tabular}{lllll}
\hline Type de forêts & Densité $\left(\right.$ tige $\left.\cdot \mathrm{ha}^{-1}\right)$ & Surface $(\mathrm{ha})$ & Localité & Source \\
\hline Denses humides & 0,38 & $198653(\mathrm{~g})$ & Sud-est du Gabon & CEB, 2015 \\
sempervirentes & 0,14 & 198734 & & CEB, 2015 \\
& 0,04 & 199436 & & CEB, 2015 \\
\cline { 2 - 4 } & 0,50 & 273984 & Nord-ouest du Gabon & ROUGIER, 2001 \\
\cline { 2 - 4 } & 0,40 & $273545(\mathrm{f})$ & Nord-est du Gabon & ROUGIER, 2002 \\
& 0,30 & $154456(\mathrm{e})$ & & OLAM, 2009 \\
\cline { 2 - 4 } & 0,16 & $79886(\mathrm{a})$ & Sud du Cameroun & WIJMA, 2011 \\
\hline Semi-décidues & 0,13 & 42810 & & WIJMA, 2011 \\
& 0,98 & $67500(\mathrm{~b})$ & Est du Cameroun & SFID, 2005 \\
& 0,51 & $176071(\mathrm{c})$ & & PALLISCO, 2004 \\
& 0,87 & $47585(\mathrm{~d})$ & & PALLISCO, 2004 \\
\hline
\end{tabular}

Les lettres a, b, c, d, e, f et g correspondent aux graphiques de la figure 4 - the letters $a, b, c, d, e, f$, and $g$ correspond to the graphs in figure 4 . 


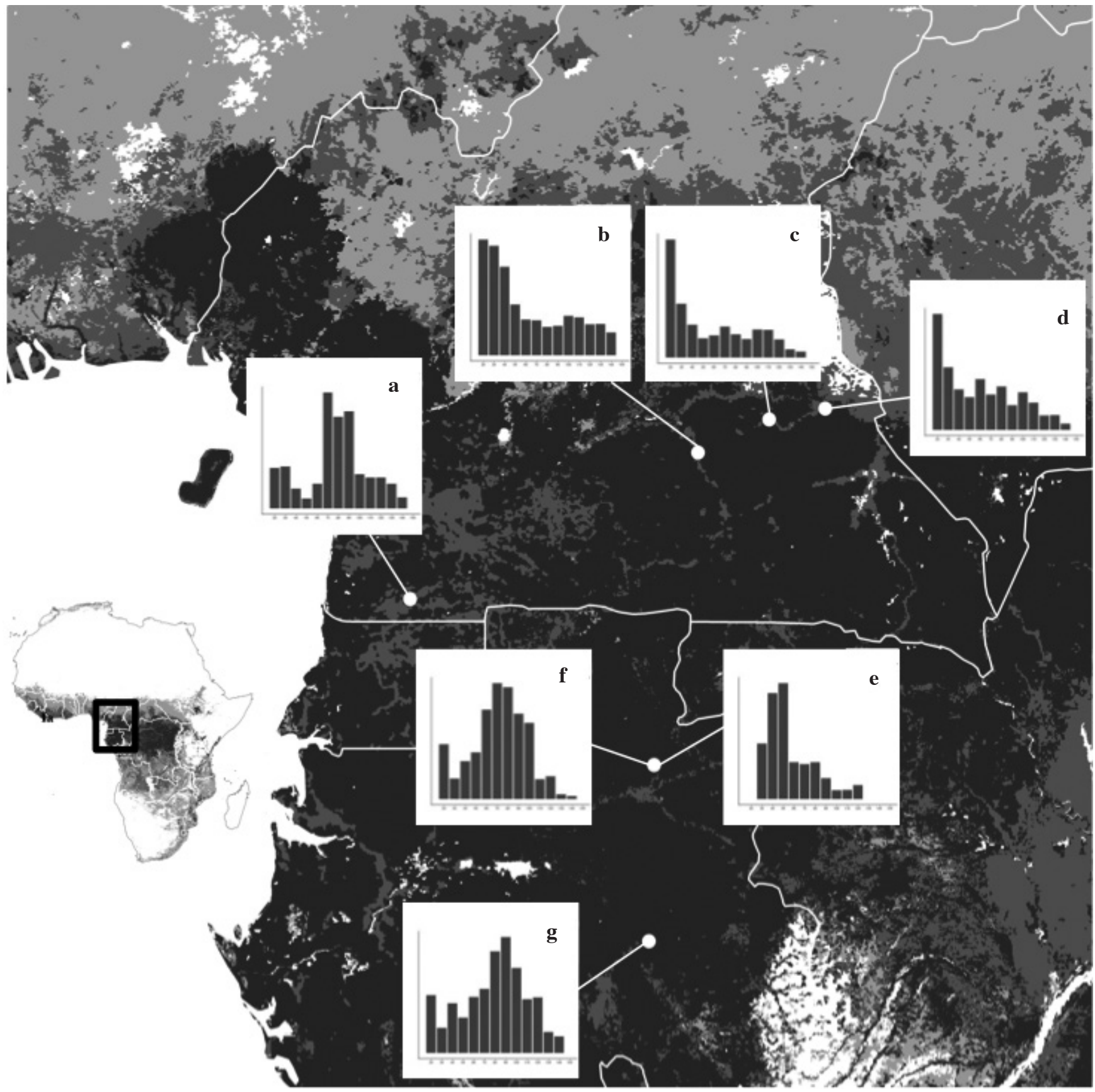

Figure 4. Représentation schématique sur base de données réelles des structures diamétriques de Cylicodiscus gabunensis au sud du Cameroun (a : 42810 ha), à l'est du Cameroun (b : 67500 ha, c : 48042 ha et d : 47170 ha), au nord-est du Gabon (e : 154456 ha et $\mathbf{f}: 273545$ ha) et au sud-est du Gabon (g : 198653 ha) - Schematic representation based on real data of diametric structures of Cylicodiscus gabunensis in Southern Cameroon (a: 42,810 ha), Eastern Cameroon (b: 67,500 ha, c: 48,042 ha and $\boldsymbol{d}:$ 47,170 ha), Northeastern Gabon (e: 154,456 ha and f: 273,545 ha) and Southeastern Gabon (g: 198,653 ha).

Pour chaque structure diamétrique : (i) l'axe des abscisses présente 14 classes de diamètre ([20-30[, [30-40[, ..., [140-150[ et $\geq 150 \mathrm{~cm}$ ), (ii) l'axe des ordonnées représente les proportions $(\%)$ des effectifs par classe de diamètre - For each diametric structure: $(i)$ the abscissa axis has 14 classes of the diameter classes ([20-30[, [30-40[, ..., [140-150[ and $\geq 150 \mathrm{~cm}$ ), (ii) the ordinate axis represents the proportions (\%) of the stem numbers by diameter class.

les deux types forestiers. Il est premièrement possible que les forêts étudiées ne soient pas au même stade de succession. Cette hypothèse est toutefois peu probable, la plupart des forêts inventoriées étant décrites comme des forêts secondaires âgées (références mentionnées dans le tableau 1). Bien que certaines aient été 
soumises en partie à une exploitation ultérieure, il est peu probable que celle-ci ait influencé les structures de populations, car les différentes concessions ont globalement les mêmes historiques d'exploitation et les mêmes intensités de prélèvement. Par ailleurs, lors de l'élaboration des plans d'aménagement, $C$. gabunensis n'était pas encore exploité. Enfin, on pourrait avancer une hypothèse relative à des perturbations naturelles ou anthropiques plus récentes au sein de la forêt camerounaise. Cette assertion est néanmoins peu vraisemblable puisque Morin-Rivat et al. (2017) obtiennent des structures de populations en cloche pour les espèces étudiées. Les raisons expliquant de telles différences de structures demeurent donc énigmatiques et mériteraient d'être étudiées.

\subsection{Reproduction de C.gabunensis}

Phénologie. Cylicodiscus gabunensis est une espèce brièvement caducifoliée (Meunier et al., 2015). Sa phénologie foliaire au nord-est du Gabon (Makokou) a été décrite par Hecketsweiler (1992). La perte des feuilles a lieu durant la petite saison sèche (janvierfévrier). La floraison est observée de novembre à avril couvrant en partie les saisons pluvieuses (septembredécembre et mars-mai) et la petite saison sèche. La période de maturation des fruits est peu décrite dans la littérature mais semble assez longue dans la mesure où les fruits matures s'observent seulement à partir de septembre-octobre (saison pluvieuse), juxtaposant ainsi la période de floraison (Gautier-Hion et al., 1985 ; Hecketsweiler, 1992; White \& Abernethy, 1996 ; Ayarkwa \& Owusu, 2008). En Côte d'Ivoire, la floraison de $C$. gabunensis a lieu en mars en fin de grande saison sèche et les arbres portent les fruits matures entre novembre (fin de la petite saison des pluies) et janvier (grande saison sèche) (voire février - mars de l'année suivante pour les individus tardifs ; Aubréville, 1959 ; De La Mensbruge, 1966). Ce patron de reproduction est quasiment le même dans d'autres pays d'Afrique de l'Ouest: Ghana et Nigéria (Irvine, 1961 ; Hawthorne, 1995; Ayarkwa \& Owusu, 2008). En définitive, de l'Afrique de l'Ouest jusqu'au Gabon, la reproduction de C. gabunensis à l'échelle des populations parait donc assez similaire. À notre connaissance, les diamètres de floraison et de fructification (Ouédraogo et al., 2018) n'ont pas encore été déterminés pour C.gabunensis, bien que des semenciers de diamètre $(d h p)$ supérieur à $60 \mathrm{~cm}$ aient été observés (Grogan \& Jurandir, 2006). De telles informations sont cruciales en matière de sylviculture et d'aménagement forestier.

\section{Pollinisation, dispersion de graines et germination.} La pollinisation chez C.gabunensis n'est pas documentée. Toutefois, la présence d'un disque dans la structure florale de l'espèce suggèrerait l'entomophilie.
Considérant que les graines sont ailées sur tout leur pourtour (diaspores à appendices saliformes), l'espèce est dite ptérochore avec une dispersion anémochore (Doucet, 2003 ; Meunier et al., 2015).

Plusieurs facteurs pourraient nuire à la germination, notamment :

- la difficulté des graines à se détacher de la valve du fruit mature lors de l'ouverture de la gousse, cette gousse pouvant tomber avec des graines encore attachées (De La Mensbruge, 1966) ;

- la consommation des fruits immatures par les primates (White \& Abernethy, 1996; MangamaKoumba et al., 2016) ;

- les attaques des larves d'insectes minant les graines

(Doucet, communication personnelle).

Une meilleure connaissance de ces facteurs constituerait un levier pour une meilleure connaissance de l'espèce.

La plantule (Figure 5) se caractérise par un hypocotyle court $(1 \mathrm{~cm})$, des cotylédons très cordés restant généralement enfermés dans leur enveloppe (ou s'en libérant tardivement, figure 5), un épicotyle glabre et long de 5 à $10 \mathrm{~cm}$ (De La Mensbruge, 1966). Toutefois, l'absence de feuilles opposées suggérerait que l'épicotyle serait moins développé (Wieringa, 1999). Les premières feuilles sont alternes, composées, simplement pennées et comportent deux paires de folioles avec parfois une foliole terminale. Les folioles sont de forme elliptique-ovée (2 à 2,5 x 1 à $1,5 \mathrm{~cm}$ ) à base obtuse asymétrique et au sommet acuminé. Le pétiole mesure 1 à $2 \mathrm{~cm}$. De petites pointes stipellaires rapidement caduques sont présentes (De La Mensbruge, 1966). Issembé (2007) confirme que la germination est de type cryptocotyle (cotylédons enfermés dans les téguments) et semi-hypogée (hypocotyle court). Les racines des plantules sont souvent recouvertes de mycorhizes ectotrophes sous forme de nombreuses

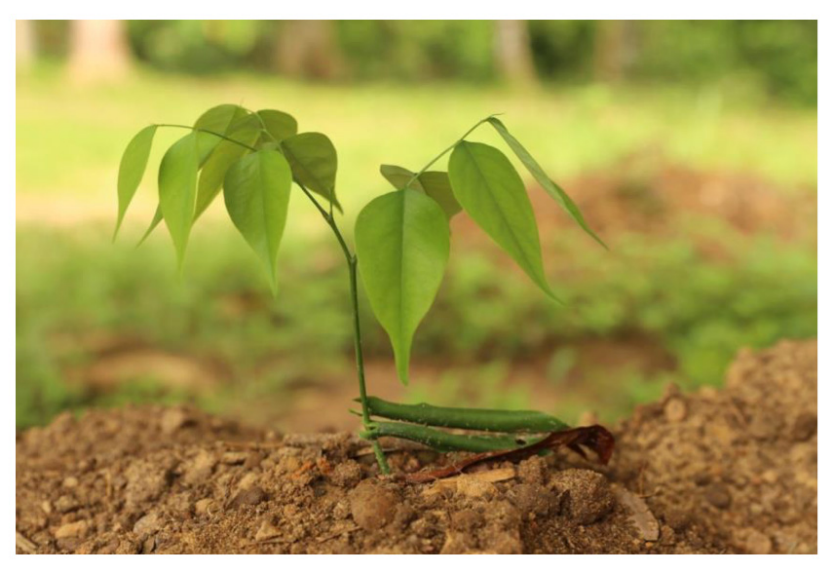

Figure 5. Plantule de Cylicodiscus gabunensis - Seedling of Cylicodiscus gabunensis () JL. Doucet. 
petites aspérités durant une période relativement courte (De la Mensbruge, 1966). Ces mycorhizes ectotrophes disparaissent probablement et font place à des endomycorhizes arbusculaires (Onguene \& Kuyper, 2001).

\section{SYLVICULTURE ET AMÉNAGEMENT FORESTIER}

\subsection{Sylviculture}

Éducation en pépinière et croissance en plantations. Les graines dont la masse est d'environ $0,1 \mathrm{~g}$ ne présentent pas de dormance (De la Mensbruge, 1966). En pépinière, le taux de germination de C. gabunensis est élevé et atteint $70 \%$ (De la Mensbruge, 1966 ; Nimbot, 2005). La germination des graines est très rapide et démarre cinq jours après le semis. L'échelonnement des levées s'effectue sur 28 jours. Les plantules atteignent une hauteur de $40 \mathrm{~cm}$ en huit mois (Nimbot, 2005). À notre connaissance, les essais en plantation de $C$.gabunensis sont rares.

Doucet et al. (2016) ont introduit l'espèce dans des plantations multispécifiques (par sous-placettes de 25 plants) dans le sud-est du Cameroun. Ces plantations ont été réalisées dans des zones de forêts dégradées où le sous-bois a été éliminé à la machette et seuls quelques gros arbres ont été conservés. Sous un ombrage modéré, la croissance moyenne en diamètre (après 690 jours d'observation) a été de $5,1 \mathrm{~mm} \cdot \mathrm{an}^{-1}$ (effectif suivi : $\mathrm{n}=102$; écart-type de $0,8 \mathrm{~mm}$ ) et le taux de survie a atteint $83 \%$. Les données disponibles sur la croissance en hauteur proviennent aussi du Cameroun (Daïnou \& Doucet, communication personnelle). En forêt sempervirente au sud-ouest du pays, des plantations non entretenues de quatre à six ans établies sur des parcs à grumes abandonnés ( $\sim 500 \mathrm{~m}^{2}$ par parc ; sol compacté) affichent une survie de $63 \%$ et une croissance moyenne en hauteur de $102 \mathrm{~cm} \cdot \mathrm{an}^{-1}(\mathrm{n}=71)$. À l'est du pays dans des forêts de transition, des plants d'âges similaires installés en plantations multispécifiques dans de larges zones dégradées ( 1 ha ; sol non compacté ; milieu plus ouvert) croissent annuellement en hauteur de $43 \mathrm{~cm} \cdot \mathrm{an}^{-1}$ et le taux de survie après cinq ans est de $74 \%(n=108)$. Il semble donc que la croissance soit très variable en fonction des conditions écologiques, ce qui confirme la nécessité de procéder à une évaluation plus rigoureuse des besoins en lumière et en eau des plantules de C. gabunensis, voire de tester à terme les performances par provenance géographique.

Croissance et mortalité des arbres adultes. Les études de croissance de $C$. gabunensis sont rares ou en cours de réalisation dans certains pays de l'aire de distribution (notamment au Cameroun et au Gabon). Selon l'Office National de Développement des Forêts du Cameroun (ONADEF, 1992), l'accroissement moyen en diamètre serait de $0,4 \mathrm{~cm} \cdot \mathrm{an}^{-1}$. Toutefois, aucune donnée n'est disponible sur la façon dont cet accroissement a été calculé. Selon les données issues du projet DynAfFor (Cirad, 2014), les valeurs d'accroissement diamétrique moyen de $C$. gabunensis (individus dominants dont les diamètres sont compris entre 70 et $89,9 \mathrm{~cm}$, effectif compris entre 21 et 31 ) varient selon les types de forêts, probablement en lien avec les conditions climatiques et édaphiques. À l'ouest du Cameroun, elles seraient significativement plus élevées $-0,87 \pm 0,15 \mathrm{~cm} \cdot \mathrm{an}^{-1}$ (IC95\%) et 0,86 $\pm 0,29 \mathrm{~cm} \cdot \mathrm{an}^{-1}(\mathrm{IC} 95 \%)$ respectivement à Ma' an et Mamfe - que celles observées à Lastoursville au Gabon : 0,51 $\pm 0,03\left(\right.$ IC95\%) $\mathrm{cm} \cdot \mathrm{an}^{-1}$ (Tableau 2). Picard \& Gourlet-Fleury (2011), en compilant plusieurs sources, suggèrent un accroissement annuel moyen (toutes classes de diamètres confondues) de $0,5 \mathrm{~cm} \cdot \mathrm{an}^{-1}$ en forêt non exploitée.

La mortalité naturelle chez cette essence n'a quasiment jamais été étudiée (Picard \& Gourlet-Fleury, 2011). Les données préliminaires issues des dispositifs de

Tableau 2. Synthèse des données d'accroissement diamétrique annuel moyen (AAM) de Cylicodiscus gabunensis et caractéristiques des sites d'observation (coordonnées, climat selon Köppen, température et précipitations moyennes) - Annual diameter increment for Cylicodiscus gabunensis and characteristics of the observation sites (coordinates, climate according to Köppen, temperature and average precipitation), www.climate-data.org

\begin{tabular}{|c|c|c|c|c|c|}
\hline $\begin{array}{l}\text { AAM }\left(\mathrm{cm} \cdot \mathrm{an}^{-1}\right) \text { et } \\
\text { intervalle de confiance }\end{array}$ & $\begin{array}{l}\text { Nombre } \\
\text { d'arbres }\end{array}$ & $\begin{array}{l}\text { Gamme } \\
\text { de diamètre }(\mathrm{cm})\end{array}$ & $\begin{array}{l}\text { Temps } \\
\text { d'observation (année) }\end{array}$ & Zone d'étude & Source \\
\hline $0,87 \pm 0,15$ & 31 & 50 à 70 & 4 & $\begin{array}{l}\text { Cameroun (Ma'an) } \\
2^{\circ} 21^{\prime} \mathrm{N}, 10^{\circ} 37^{\prime} \mathrm{E} \\
\mathrm{Am}, 23,3^{\circ} \mathrm{C}, 2073 \mathrm{~mm}\end{array}$ & Cirad, 2014 \\
\hline $0,86 \pm 0,29$ & 37 & 70 à 90 & 4 & $\begin{array}{l}\text { Cameroun (Mamfe) } \\
5^{\circ} 46^{\circ} \mathrm{N}, 917^{\prime} \mathrm{E} \\
\mathrm{Am}, 26,7^{\circ} \mathrm{C}, 2753 \mathrm{~mm}\end{array}$ & Cirad, 2014 \\
\hline $0,5 \pm 0,03$ & 20 & 70 à 90 & 3 & $\begin{array}{l}\text { Gabon (Lastoursville) } \\
0^{\circ} 49^{\prime} \mathrm{S} ; 12^{\circ} 42^{\prime} \mathrm{E} \\
\text { Aw, } 25,5^{\circ} \mathrm{C}, 1622 \mathrm{~mm}\end{array}$ & $\begin{array}{l}\text { Doucet } \\
\text { et al., } 2007\end{array}$ \\
\hline
\end{tabular}


DynAfFor récemment installés au Cameroun estiment, toutes classes diamétriques confondues, un taux annuel de mortalité à 0,17 - 0,33\% après un suivi sur cinq ans, des valeurs à confirmer sur une période beaucoup plus longue.

\subsection{Aménagement forestier}

Tarif de cubage. La connaissance du volume des arbres est cruciale tant pour des usages commerciaux, que pour la gestion des massifs forestiers (Lanly, 1965). En toute rigueur, les formules permettant d'estimer les volumes, encore appelées tarifs de cubage, devraient être spécifiques et valides uniquement pour des localités aux conditions environnementales similaires (Rondeux, 1999). Au Cameroun, l'ONADEF (1992) impose l'utilisation de tarifs de cubage en fonction de quatre zones géographiques appelées phases d'inventaires. Toutefois, Fayolle et al. (2013) et Ligot et al. (2018) montrent que ces tarifs sont biaisés et sous-estiment les volumes réels. Ligot et al. (2018) ont dès lors élaboré un nouveau tarif de cubage pour C. gabunensis à partir d'individus étudiés principalement dans deux sites: Djoum, dont la forêt est de transition entre les types sempervirents et semi-décidus et Mindourou, dont la forêt est semi-décidue (n pieds $=89$, diamètre compris entre 31 et $175 \mathrm{~cm}$ ). Ils préconisent d'utiliser la formule suivante pour le calcul du volume exploitable de la bille sur écorce $\left(\mathrm{V}\right.$, en $\left.\mathrm{m}^{3}\right)$ en fonction du diamètre de référence $(\mathrm{D}$, en $\mathrm{cm})$

$$
V=-0,00794 \times D+0,001194 \times D^{2} .
$$

Picard \& Gourlet-Fleury (2011) synthétisent les autres équations disponibles pour C.gabunensis, dont celle d'Akindele (2005) élaborée au Nigeria. Ces formules sont moins fiables que la formule précédente car reposant sur des effectifs réduits $(\mathrm{n}=15$ dans l'étude d'Akindele, $2005 ; V=0,0102+0,000166 \times D^{1,79} \times$ $H^{0,822}$, avec $V$, en $\mathrm{m}^{3} ; D$, en $\mathrm{cm}$ et $H$, en $\mathrm{m}$ ) ou sur des groupes d'espèces.

Normes et règles d'exploitation. Des mesures légales concernent directement l'exploitation de C.gabunensis en Afrique centrale. La première est le diamètre minimum d'exploitabilité, communément appelé DME. Il correspond au diamètre à $1,30 \mathrm{~m}$ du sol $(d h p)$ ou au-dessus des contreforts, à partir duquel un arbre peut être abattu. C'est un paramètre capital pour la gestion. $\mathrm{Ce}$ DME varie selon les pays. Au Cameroun, le DME de $C$.gabunensis est de $60 \mathrm{~cm}$ (ONADEF, 1992), tout comme en République du Congo (Décret n²002-437 du 31 décembre 2002). Par contre, il est de $70 \mathrm{~cm}$ au Gabon (Loi n 16/01 du 31 décembre 2001). La seconde norme commune à tous les pays d'Afrique centrale repose sur le taux minimum de reconstitution des stocks exploités (reconstitution entre deux passages successifs de l'exploitation au même endroit). Ce taux représente la part de population (en effectifs au-dessus du DME) qui sera reconstituée au bout d'une rotation. Au Cameroun par exemple, ce taux ne doit pas être inférieur à $50 \%$ pour les quatre classes supérieures au DME ([60-69,9 cm] ; ([70-79,9 cm]; [80-89,9 cm] et [90-99,9 cm]) après une période de rotation de 30 ans (révision de l'Arrêté

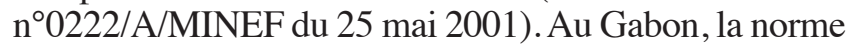
est d'un taux de reconstitution minimum de $40 \%$ (tous les effectifs au-dessus du DME) après une rotation minimum de 20 ans (Loi n 16/01 du 31 décembre 2001).

Ces exigences légales constituent une avancée significative dans la gestion des essences exploitées en Afrique centrale, mais elles souffrent encore d'importantes lacunes :

- le minimum de reconstitution imposé concerne les effectifs et ne garantit nullement le renouvellement d'une fraction notable des volumes exploités ;

- les valeurs seuils de reconstitution imposées dans ces pays n'atteignent pas les $100 \%$;

- les DME ont été fixés sans étude préalable des diamètres de fructification régulière.

Ainsi, au bout de plusieurs rotations, cela peut conduire à une raréfaction considérable de certaines espèces (Karsenty \& Gourlet-Fleury, 2006).

\section{COMMERCE DU BOIS}

L'espèce ne fait l'objet d'aucune restriction d'exportation dans le cadre de la CITES ${ }^{1}$ et n'est pas reprise sur la liste rouge de l'UICN (IUCN, 2019). Le bois de C. gabunensis occupe une place de choix dans l'industrie du bois. Il est commercialisé principalement sous le nom d'okan ou Denya (CTFT, 1955; ATIBT, 2010). En 2009, le volume total exporté (grumes et sciages) de l'Afrique (Cameroun, République du Congo, Gabon, Ghana) s'élevait à $367942 \mathrm{~m}^{3}$ (ATIBT, 2010). Avant l'arrêt des exportations de grumes en 2010, le Gabon était le principal exportateur de grumes de C.gabunensis avec $190949 \mathrm{~m}^{3}$ par an en moyenne entre 2007 et 2009 (ATIBT, 2010). Ces exportations hissaient l'essence au rang de second bois le plus exporté en grume après l'okoumé (Aucoumea klaineana Pierre) (ATIBT, 2010). Au Cameroun, le bois de l'okan occupait la seconde place des exportations de grumes en 2010 et 2011 (ATIBT, 2012). En 2016, l'okan était la sixième espèce la plus produite en Afrique centrale (Figure 6) (FRM, 2018).

\footnotetext{
${ }^{1}$ CITES, Convention On International Trade in Endangered Species of Wild Fauna and Flora, www.cites. org, (16 mars 2019).
} 


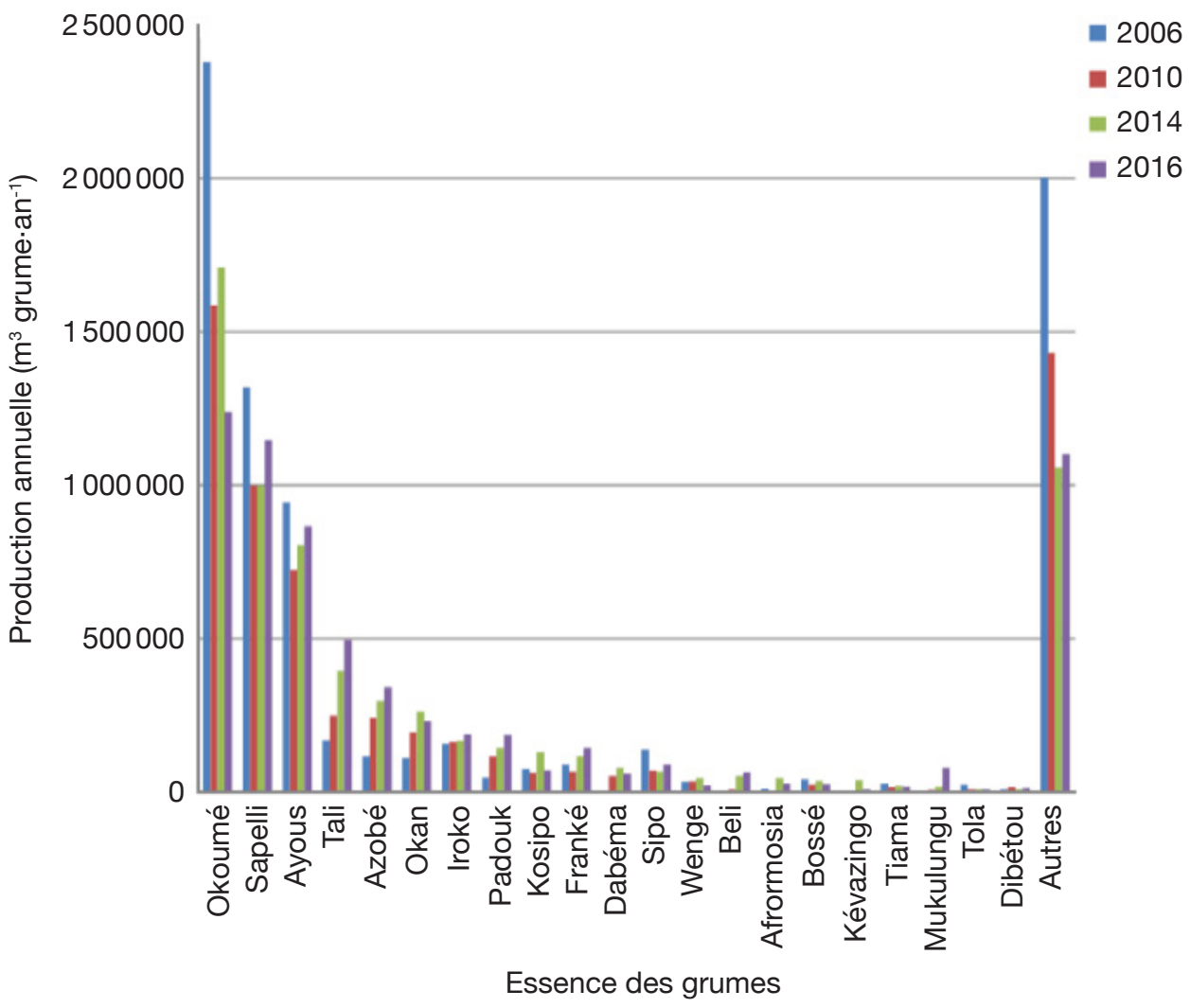

Figure 6. Évolution des productions annuelles de grumes par essence dans le bassin du Congo (hors Guinée équatoriale) - Evolution of annual log production by species in the Congo Basin (excluding Equatorial Guinea) (FRM, 2018).

tableau 3 présente les principales caractéristiques technologiques du bois de cette espèce. La couleur du bois varie de brun jaunâtre à brun doré. Le bois est contrasté d'une fine coloration verdâtre lorsqu'il est exposé à la lumière. L'aubier épais est rose grisâtre et bien différencié. Son grain est moyen à grossier et présente un contrefil léger (parfois prononcé) et de fines mailles. $\mathrm{Ce}$ bois nécessite un séchage lent. Il est très durable (Vivien \& Faure, 1985; ATIBT, 1986 ; Gérard et al., 2011). Il résiste aux champignons, térébrants marins, termites et xylophages du bois sec (CTFT, 1955 ; Déon et al., 1980 ; Ayarkwa \& Owusu, 2008). Le bois de $C$. gabunensis couvre la classe d'emploi 4 et 5 , c'est-à-dire qu'il est adapté aux conditions

La Chine, Hong-Kong et Taiwan sont les plus gros importateurs en absorbant $90 \%$ de la production (équivalents bois ronds). Viennent ensuite les pays suivants: Belgique, France, Pays-Bas, Vietnam, Inde, Ukraine, Italie et Portugal (ATIBT, 2012).

FRM (2018) estiment que le volume net d'okan mobilisable par année serait compris entre 290000 et $390000 \mathrm{~m}^{3}$. Les réserves les plus importantes sont situées au Gabon (50 à $75 \%$ de ce volume), au Cameroun (25 à $50 \%$ ) et dans le sud de la République du Congo (10 à $25 \%)$.

Le succès du bois de $C$.gabunensis sur le marché international des bois tropicaux est lié aux qualités technologiques de son bois qui pourraient offrir de multiples usages industriels à ses utilisateurs (CTFT, 1955).

\section{USAGES}

\subsection{Le bois}

L'anatomie du bois de $C$.gabunensis a déjà fait l'objet d'une description relativement bien détaillée par Hédin (1929), le CTFT (1955) et N.P. Mollel, P. Détienne \& E.A. Wheeler in Ayarkwa \& Owusu (2008). Le d'humidité permanente, immergé dans le sol, l'eau de mer, les milieux marins y compris les eaux saumâtres des estuaires (CTFT, 1955 ; Vernay \& Fouquet, 2000 ; Meunier et al., 2015). Le bois est utilisé pour la construction des ponts en milieu marin, la production des traverses de chemin de fer, la menuiserie extérieure, les travaux hydrauliques, le platelage, les vannes d'écluse, la parqueterie lourde, la charronnerie, les étais de mines et la sculpture (CTFT, 1955; De Saint Aubin, 1963 ; ATIBT, 1986 ; Ayarkwa \& Owusu, 2008 ; Gérard et al., 2011 ; Meunier et al., 2015).

\subsection{Usages traditionnels et composés chimiques de C.gabunensis}

Cylicodiscus gabunensis est utilisé par les communautés rurales pour diverses raisons. Il est considéré comme arbre fétiche notamment par certains peuples autochtones, dont les Bavungu et Eshiras du Gabon, chez qui l'arbre occupe une place centrale dans les rites et traditions (Villiers, 1989 ; Quiroz \& van Andel, 2015). L'arbre peut servir d'ombrage dans les jardins de cases ou dans les jachères (Boldrini et al., 2015) et le bois peut être utilisé pour produire du charbon. Son écorce est utilisée comme savon pour le nettoyage et comme poison de pêche. Les feuilles sont utilisées 
Tableau 3. Caractéristiques technologiques et classes d'emploi du bois de Cylicodiscus gabunensis - Technological features and work properties of Cylicodiscus gabunensis wood.

\begin{tabular}{|c|c|c|c|}
\hline Propriété & Condition de mesures & $\begin{array}{l}\text { Valeur des caractéristiques } \\
\text { technologiques }\end{array}$ & Source \\
\hline Densité du bois $\left(\mathrm{g} \cdot \mathrm{cm}^{-3}\right)$ & Bois demi-sec & 1,11 & Hédin, 1929 \\
\hline Densité du bois $\left(\mathrm{g} \cdot \mathrm{cm}^{-3}\right)$ & $\begin{array}{l}12 \% \text { d'humidité, } \\
1 \mathrm{Mpa}=1 \mathrm{~N} / \mathrm{mm}^{2}\end{array}$ & $0,91 \pm 0,10 \sigma$ & Gérard et al., 2011 \\
\hline Dureté monnin & $\begin{array}{l}12 \% \text { d'humidité, } \\
1 \mathrm{Mpa}=1 \mathrm{~N} / \mathrm{mm}^{2}\end{array}$ & $10,3 \pm 3,40 \% \sigma$ & Gérard et al., 2011 \\
\hline Rétractibilité (\%) & $\begin{array}{l}\text { Volumique } \\
\text { Tangentielle } \\
\text { Radiale }\end{array}$ & $\begin{array}{l}0,61 \pm 0,10 \% \sigma \\
7,9 \pm 1 \% \sigma \\
5,8 \pm 0,60 \% \sigma\end{array}$ & Gérard et al., 2011 \\
\hline Contrainte de rupture en compression (Mpa) & $\begin{array}{l}12 \% \text { d'humidité, } \\
1 \mathrm{Mpa}=1 \mathrm{~N} / \mathrm{mm}^{2}\end{array}$ & $82 \pm 12 \% \sigma$ & Gérard et al., 2011 \\
\hline Contrainte de rupture en flexion statique (Mpa) & $\begin{array}{l}12 \% \text { d'humidité, } \\
1 \mathrm{Mpa}=1 \mathrm{~N} / \mathrm{mm}^{2}\end{array}$ & $134 \pm 23 \% \sigma$ & Gérard et al., 2011 \\
\hline Module d'élasticité longitudinal (Mpa) & $\begin{array}{l}12 \% \text { d'humidité, } \\
1 \mathrm{Mpa}=1 \mathrm{~N} / \mathrm{mm}^{2}\end{array}$ & $22260 \pm 3348 \% \sigma$ & Gérard et al., 2011 \\
\hline Point de saturation des fibres & & $25 \%$ & Gérard et al., 2011 \\
\hline Conductivité thermique $(\lambda)(\mathrm{W} /[\mathrm{m} . \mathrm{K}])$ & & 0,29 & Gérard et al., 2016 \\
\hline
\end{tabular}

Tableau 4. Principales utilisations traditionnelles de Cylicodiscus gabunensis en Afrique - Main traditional uses of Cylicodiscus gabunensis in Africa.

\begin{tabular}{|c|c|c|c|}
\hline Maladies et symptômes soignés & Partie de la plante utilisée & Modes de préparation & Sources \\
\hline $\begin{array}{l}\text { Maux de ventre, maux d'estomac, } \\
\text { prostatite, rhumatisme, diabète, } \\
\text { abcès interne, fièvre, maladies } \\
\text { vénériennes, paludisme ou malaria, } \\
\text { psoriasis, rhumatisme, antalgique, } \\
\text { anti-vomitif }\end{array}$ & Écorce & Décoction & $\begin{array}{l}\text { Villiers, } 1989 \text {; Betti, } \\
2004 \text {; Ayarkwa \& } \\
\text { Owusu, } 2008 \text {; Din } \\
\text { et al., } 2011 \text {; Ndah } \\
\text { et al., } 2013 \text {; Nga et al., } \\
2016\end{array}$ \\
\hline Stérilité & Écorce & $\begin{array}{l}\text { Décoction avec du vin; } \\
\text { Décoction avec de l'eau } \\
\text { de mer et du jus de Costus } \\
\text { lucanusianus J. Braun \& K. } \\
\text { Schum }\end{array}$ & Angone et al., 2009 \\
\hline Infections respiratoires & Écorce & $\begin{array}{l}\text { Décoction et Coula edulis } \\
\text { Baill. et Garcinia kola } \\
\text { Heckel }\end{array}$ & Mpondo et al., 2017 \\
\hline Varicelle & Écorce & Macération & Nga et al., 2016 \\
\hline Rougeole & Écorce & $\begin{array}{l}\text { Macération avec le vin de } \\
\text { palme (Elaeis guineensis } \\
\text { Jacq.) }\end{array}$ & Nga et al., 2016 \\
\hline Diabète & Écorce & Infusion & Din et al., 2011 \\
\hline
\end{tabular}

comme fourrage pour les animaux (Villiers, 1989; Ayarkwa \& Owusu, 2008). Cylicodiscus gabunensis doit sa notoriété en pharmacopée traditionnelle aux nombreuses vertus qui lui sont reconnues (Tableau 4 ;
Okokon et al., 2006; Ayarkwa \& Owusu, 2008). L'écorce est utilisée en lavement intestinal pour guérir la diarrhée, le rhumatisme, les abcès ou servir d'antimicrobien (Kouitcheu et al., 2006 ; Okokon et al., 
2006 ; Ndah et al., 2013). Combinée à l'écorce d'autres espèces, les champs d'application en pharmacopée traditionnelle sont encore plus larges. Par exemple, $\mathrm{Nga}$ et al. (2016) signalent qu'une décoction d'écorce d'Oboto (Mammea africana Sabine) et de C. gabunensis est utilisée en lavement intestinal au Cameroun dans le traitement des chlamydias. Les feuilles macérées sont aussi utilisées dans le traitement de la migraine (Villiers, 1989 ; Ayarkwa \& Owusu, 2008).

Les études phytochimiques, notamment de l'écorce de $C$.gabunensis, ont permis de mettre en évidence l'existence de plusieurs composés utiles. Kouitcheu et al. $(2006,2007)$ ont démontré que les extraits d'acétate d'éthyle de $C$.gabunensis sont fournis en saponines, des molécules complexes aux propriétés tensioactives qui font mousser leurs solutions et servent de détergent, ce qui expliquerait son usage comme savon. Ces auteurs ont montré également que les extraits renfermaient des tannins, polyphénols, coumarines, stérols, triterpènes, sucres réducteurs et flavonoïdes qui confèrent à cette espèce des propriétés antimicrobiennes (notamment contre Staphylococcus aureus, Proteus vulgaris et Baccillus cereus T.) et antidiarrhéiques. Aldulaimi et al. (2017) ont montré que les acides phénoliques (gallate d'éthyle et les acides benzoïques) présents dans l'écorce confèrent à $C$. gabunensis des propriétés antipaludéennes. En considérant les nombreux symptômes traités en utilisant différentes parties de l'arbre en pharmacopée traditionnelle, il existerait potentiellement de nombreux principes actifs que des investigations futures pourraient révéler.

\section{CONCLUSIONS ET PERSPECTIVES}

Cette synthèse bibliographique a dressé l'état des connaissances actuelles sur C.gabunensis. L'espèce semble être bien documentée en termes de description botanique, d'anatomie du bois et d'ethnobotanique. Par contre, les facteurs expliquant les structures actuelles des populations demeurent à élucider en lien avec les exigences écologiques de l'espèce, dont les besoins en lumière et en fertilité des sols. Des études archéobotaniques et des dispositifs en conditions contrôlées seraient particulièrement utiles afin de mettre en place une stratégie de régénération adaptée à l'espèce. D'autres aspects méritent des investigations approfondies afin d'assoir de véritables politiques de gestion durable de l'espèce, dont sa phénologie, sa croissance et les facteurs intervenant dans la régénération (prédation et parasitisme). Un diamètre minimum d'exploitation de $60 \mathrm{~cm}$, tel qu'actuellement appliqué au Cameroun, est probablement trop faible, mais cela doit être confirmé par des études phénologiques. L'ensemble de ces informations devraient permettre de mettre en place des techniques sylvicoles efficientes qui permettraient le maintien de $C$.gabunensis sur le long terme.

\section{Remerciements}

Les auteurs remercient chaleureusement, pour le financement des activités de recherche, le Fonds Français pour l'Environnement Mondial, au titre des conventions

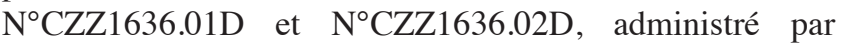
l'Agence Française de Développement dans le cadre du projet DynAfFor, mis en œuvre par le consortium ATIBT/ CIRAD/Nature ${ }^{+} /$Gembloux Agro-Bio Tech. Ils remercient pour la même raison l'entreprise CEB-Precious Woods et la Precious Forest Foundation. Plusieurs entreprises ont accepté de nous communiquer les informations relatives aux structures de populations. Nous remercions particulièrement PALLISCO, CEB-Precious Woods, ROUGIER et WIJMA. Nous remercions enfin Nature $^{+}$et l'équipe de la CARE Forest is life du centre de recherche TERRA de Gembloux Agro-Bio Tech (Université de Liège) pour les divers appuis apportés.

\section{Bibliographie}

Agyeman V., Swaine M. \& Thompson J., 1999. Responses of tropical forest tree seedlings to irradiance and the derivation of a light response index. J. Ecol., 87, 815827.

Akindele S.O., 2005. Volume functions for common timber species of Nigeria's forests - a technical document. Vancouver, Canada: University of British Columbia; Akure, Nigeria: Federal University of Technology.

Aldulaimi O. et al., 2017. A characterization of the antimalarial activity of the bark of Cylicodiscus gabunensis Harms. J. Ethnopharmacol., 198, 221225.

Angone S.A. et al., 2009. Quelques plantes utilisées en médecine traditionnelle pour le traitement de la stérilité chez les femmes au Gabon. Ethnopharmacologia, 43, 52-58.

ATIBT (Association Technique Internationale des Bois Tropicaux), 1986. Atlas des bois tropicaux. Tome I. Afrique. Paris : ATIBT.

ATIBT (Association Technique Internationale des Bois Tropicaux), 2010. La lettre de l'ATIBT $n^{\circ} 32$ : statistiques 2009. Paris : ATIBT.

ATIBT (Association Technique Internationale des Bois Tropicaux), 2012. La lettre de l'ATIBT $n^{\circ} 34$ : statistiques 2010. Paris : ATIBT.

Aubréville A., 1959. La flore forestière de la Côte d'Ivoire. $2^{\mathrm{e}}$ éd. Nogent-sur-Marne, France: Centre Technique Forestier Tropical.

Ayarkwa J. \& Owusu F.W., 2008. Cylicodiscus gabunensis Harms. In: Louppe D., Oteng-Amoako A.A. \& Brink M., eds. Prota 7(1): timbers/bois d'oeuvre 1. Wageningen, The Netherlands: Prota. 
Betti J.L., 2004. An ethnobotanical study of medicinal plants among the Baka pygmies in the Dja biosphere reserve, Cameroon. Afr. Stud. Monogr., 25(1), 1-27.

Biwole A., 2015. Origine et dynamique des populations d'arbres des forêts denses humides d'Afrique Centrale, le cas de Lophira alata Banks ex Gaertn C.F. (Ochnaceae). Thèse de doctorat : Gembloux Agro-Bio Tech, Université de Liège (Belgique).

Biwolé A. et al., 2015. New data on the recent history of the littoral forests of southern Cameroon: an insight into the role of historical human disturbances on the current forest composition. Plant Ecol.Evol., 148(1), 1928.

Boldrini S. et al., 2015. Approche agroforestière. Synthèse des travaux menés par le projet DACEFI 2 en agroforesterie. Document de capitalisation. Libreville : Projet DACEFI 2.

Bouchenak-Khelladi Y., Maurin O., Hurter J. \& van der Bank M., 2010. The evolutionary history and biogeography of Mimosoideae (Leguminosae): an emphasis on African acacias. Mol. Phylogenet. Evol., 57, 495-508.

Bourland N., 2013. Dynamique d'une espèce ligneuse héliophile longévive dans un monde changeant: le cas de Pericopsis elata (Harms) Meeuwen (Fabaceae) au sud-est du Cameroun. Thèse de doctorat: Gembloux Agro-Bio Tech, Université de Liège (Belgique) .

CEB, 2015.Pland'aménagement de la Concession Forestière sous Aménagement Durable de la société Precious woods -CEB 2000-2024. CEB, 2015. Libreville : TEREA.

CIRAD, 2014. Projet DYNAFFOR, dynamique des forêts d'Afrique centrale, https://www.dynaffor.org, (12/03/2019).

CJBG (Conservatoire et Jardin botaniques de la Ville de Genève), 2018. Base de données des plantes d'Afrique. Genève, Suisse : CJBG, https://www.ville-ge.ch/cjb/ bd.php, (19/03/2019).

CLIMATE-DATA.ORG. Les données climatiques pour les villes $d u$ monde entier, https://www.climate-data.org, $(30 / 03 / 2019$.

CTFT (Centre Technique Forestier Tropical), 1955. Fiches botaniques, forestières, industrielles et commerciales : okan. Bois For. Trop., 43, 11-14.

Dauby G. et al., 2016. RAINBIO : une méga-base de données des distributions de plantes vasculaires en Afrique tropicale. PhytoKeys, 74, 1-18.

De La Mensbruge G., 1966. La germination et les plantules des essences arborées de la forêt dense humide de la Côte d'Ivoire. Publication $n^{\circ} 26$. Nogent-sur-Marne, France : Centre Technique Forestier Tropical.

De Saint Aubin G., 1963. La forêt du Gabon. Publication $n^{\circ} 21$. Nogent-Sur-Marne, France: Centre Technique Forestier Tropical.

Din N. et al., 2011. Inventory and identification of plants used in the treatment of diabetes in Douala town (Cameroon). Eur. J. Med. Plants, 1(3), 60-73.
Doucet J.-L., 2003. L'alliance délicate de la gestion forestière et de la biodiversité dans les forêts du centre $d u$ Gabon. Thèse de doctorat : Faculté universitaire des Sciences agronomiques de Gembloux (Belgique).

Doucet J.-L. et al., 2007. Dynamique des peuplements forestiers d'Afrique centrale. Libreville: ENEF; Gembloux, Belgique : Faculté universitaire des Sciences agronomiques de Gembloux.

Doucet J.-L. et al., 2016. Enrichment of Central African logged forests with high-value tree species: testing a new approach to regenerating degraded forests. Int. J. Biodivers. Sci. Ecosyst. Serv. Manage., 12(1-2), 83-95.

Droissart V. et al., 2018. Beyond trees: biogeographical regionalization of tropical Africa. J. Biogeogr., 45(5), 1153-1167.

Duah-Gyamfi A. et al., 2014a. Natural regeneration dynamics of tree seedlings on skid trails and tree gaps following selective logging in a tropical moist semideciduous forest in Ghana. Open J. For., 4(1), 49.

Duah-Gyamfi A. et al., 2014b. Can harvesting for timber in tropical forest enhance timber tree regeneration? For. Ecol. Manage., 314, 26-37.

Duah-Gyamfi A. et al., 2015. Seedling abundance, composition and growth forecast under two logging intensities in a moist tropical forest in Ghana. Ghana J. For., 31, 1-20.

Engone Obiang N.L.et al.,2011.Biodiversité et perturbations anthropiques: le cas de la forêt du Haut Abanga au Gabon. In: Atelier sur la Protection des Ressources Naturelles, 14-18 février 2011, Meknès, Maroc. Meknès, Maroc : Université Moulay Ismail ; IRD.

Fayolle A. et al., 2013. Réviser les tarifs de cubage pour mieux gérer les forêts du Cameroun. Bois For. Trop., 317, 35-49.

FRM (FORET RESSOURCES MANAGEMENT), 2018. Vision stratégique et industrialisation de la filière bois dans les 6 pays du bassin du Congo, Horizon 2030. Rapport stratégique régional. Montpellier, France: FRM.

Gautier-Hion A. et al., 1985. Coadaptation entre rythmes de fructification et frugivorie en forêt tropicale humide du Gabon: mythe ou réalité. Rev. Écol. Terre Vie, 40, 405-434.

GBIF (The Global Biodiversity Information Facility), 2019. GBIF, https://www.gbif.org/what-is-gbif, (28/03/2019).

Gérard J.et al., 2011. The main technological characteristics of 245 tropical wood species (Africa). Tropix 7. Montpellier, France : CIRAD.

Gérard J., Guibal D., Paradis S. \& Cerre J.-C., 2016. Atlas des bois tropicaux : caractéristiques technologiques et utilisations. Versailles, France : Éditions Quæ.

Grogan J.\& Jurandir G., 2006. Factors limiting post-logging seedling regeneration by big-leaf Mahogany (Swietenia macrophylla) in Southeastern Amazonia, Brazil, and implications for sustainable management. Biotropica, 38(2), 219-228. 
Hawthorne W., 1995. Ecological profiles of Ghanaian forest trees. Oxford, UK: University of Oxford.

Hawthorne W. \& Gyakari N., 2006. Photoguide for the forest trees of Ghana: a tree-spotter's field guide for identifying the largest trees. Oxford, UK: University of Oxford.

Hecketsweiler P., 1992. Phénologie et saisonnalité en forêt gabonaise: l'exemple de quelques espèces ligneuses. Thèse de doctorat: Université de Montpellier II (France).

Hédin L., 1929. Note sur le bois d'Adoum (Cylicodiscus gabunensis Harms). J. Agric. Traditionnelle Bot. Appl., 95, 446-448.

Herbier National du Gabon, 2019. Plateforme de l'Herbier National du Gabon, http://vmtropicar-proto.ird.fr/gabon/ collection/collections, (06/05/2019).

Irvine F.R., 1961. Woods plants of Ghana. Oxford, UK: Oxford University Press.

Issembé Y.R., 2007. Études des stades juvéniles d'espèces ligneuses des forêts denses humides tropicales $d u$ Gabon. Mémoire de fin d'études: Faculté universitaire des Sciences agronomiques de Gembloux (Belgique).

IUCN, 2019. The IUCN Red List of Threatened Species. Version 2018-2, http://www.iucnredlist.org, (16/03/2019).

Karsenty A. \& Gourlet-Fleury S., 2006. Assessing sustainability of logging pratices in the Congo Basin's managed forest: the issue of commercial species recovery. Ecol. Soc., 11(1), 26.

Kouadio Y.L., 2009. Mesures sylvicoles en vue d'améliorer la gestion des populations d'essences forestières commerciales de l'Est du Cameroun. Thèse de doctorat : Faculté universitaire des Sciences agronomiques de Gembloux (Belgique).

Kouitcheu M.L.B. et al., 2006. Evaluation of antidiarrhoeal activity of the stem bark of Cylicodiscus gabunensis (Mimosaceae). Afr. J. Biotechnol., 5(11), 1062-1066.

Kouitcheu M.L.B. et al., 2007. Evaluation of antimicrobial activity of the stem bark of Cylicodiscus gabunensis (Mimosaceae). Afr. J. Tradit. Complement. Altern. Med., 4, 87-93.

Kryn J.M. \& Fobes E.W., 1959. The woods of Liberia. Madison, WI, USA: Forest Products Laboratory.

Kyalangalilwa B. et al., 2013. Phylogenetic position and revised classification of Acacia s.l. (Fabaceae: Mimosoideae) in Africa, including new combinations in Vachellia and Senegalia. Bot. J. Linn. Soc., 172(4), 500-523.

Lanly J.-P., 1965. Les tarifs de cubage. Bois For. Trop., 100, 19-35.

Letouzey R., 1982. Manuel de botanique forestière Afrique tropicale. Tome 2A. Nogent-sur-Marne, France : Centre technique forestier tropical.

Ligot G. et al., 2018. Réviser les tarifs de cubage pour prendre en compte l'évolution de la ressource au Cameroun. Bois For. Trop., 338, 57-71.
LPWG, 2013. Legume phylogeny and classification in the $21^{\text {st }}$ century: progress, prospects and lessons for other species-rich clades. Taxon, 62, 217-248.

LPWG, 2017. A new subfamily classification of the Leguminosae based on a taxonomically comprehensive phylogeny. Taxon, 66, 44-77.

Luckow M. \& Grimes J., 1997. A survey of anther glands in the mimosoid legume tribes Parkieae and Mimoseae. Am. J. Bot., 84, 285-297.

Luckow M., Miller J., Murphy D.J. \& Livshultz T., 2003. A phylogenetic analysis of the Mimosoideae (Leguminosae) based on chloroplast DNA sequence data. In: Klitgaard B.B. \& Bruneau A., eds. Advances in legume systematics (part 10). Kew, UK: The Royal Botanic Garden, 197-220.

Mangama-Koumba L.B. et al., 2016. Vegetarian diet in Guenon and Mangabey monkeys of Moukalaba-Doudou National Park, Gabon: similarities and differences. Int. J. Biol. Chem. Sci., 10, 2435-2446.

Manzanilla V. \& Bruneau A., 2012. Phylogeny reconstruction in the Caesalpinieae grade (Leguminosae) based on duplicated copies of the sucrose synthase gene and plastid markers. Mol. Ph. Evol., 65, 149-162.

Meunier Q., Moumbogou C. \& Doucet J.-L., 2015. Les arbres utiles du Gabon. Gembloux, Belgique: Les Presses agronomiques de Gembloux.

Morin-Rivat J., 2017. Of trees and men: new insights into man-environment relationships in the moist forests of central Africa during the late Holocene. Thèse de doctorat: Université de Liège, Gembloux Agro-Bio Tech (Belgique).

Morin-Rivat J. et al., 2017. Present-day central African forest is a legacy of the $19^{\text {th }}$ century human history. Elife, 6, e20343.

Mpondo M.E. et al., 2017. Contribution des populations des villages du centre Cameroun aux traitements traditionnels des affections des voies respiratoires. J. Anim. Plant Sci., 32, 5223-5242.

Ndah N.R. et al., 2013. Ethnobotanical study of commonly used medicinal plants of the Takamanda Rainforest South West, Cameroon. Afr. J. Plant Sci., 7, 2134.

Nga N.E. et al., 2016. Inventaire et caractérisation des plantes médicinales utilisées en thérapeutique dans le département de la Sanaga Maritime : Ndom, Ngambe et Pouma. J. Appl. Biosci., 106, 10333-10352.

Nimbot Mamba M., 2005. Étude de la germination, de la croissance et de la morphologie des plantules d'espèces ligneuses des forêts denses humides tropicales de la province de l'Ogooué-Lolo (Gabon). Mémoire de fin d'études: Faculté universitaire des Sciences agronomiques de Gembloux (Belgique).

Okokon J.E., Ita B.N. \& Udokpoh A.E., 2006. Antiplasmodial activity of Cylicodiscus gabunensis. J. Ethnopharmacol., 107, 175-178. 
OLAM, 2009. Plan d'aménagement de l'UFA de Makokou (Gabon). Libreville : TEREA.

ONADEF (Office National de Développement des Forêts du Cameroun), 1992. Normes d'études d'arbres. Yaoundé : Ministère de l'Environnement et des Forêts.

Onguene N.A. \& Kuyper T.W., 2001. Mycorrhizal associations in the rain forest of South Cameroon. For. Ecol. Manage., 140(2-3), 277-287.

Oslisly R. et al., 2013. Climatic and cultural changes in the west Congo Basin forests over the past 5000 years. Philos. Trans. R. Soc. London, Ser. B, 368, 1-11.

Ouédraogo D.Y. et al., 2018. The size at reproduction of canopy tree species in central Africa. Biotropica, 50(3), 465-476.

PALLISCO, 2004. Plan d'aménagement des UFA 10.041, 10.042 et 10.044 regroupées. Douala, Cameroun : PALLISCO.

Picard N. \& Gourlet-Fleury S., 2011. Optimisation des hypothèses et paramètres d'aménagement. Rapport de mission : PAPPFG, mission 16.

Quattrocchi U., 2012. CRC world dictionary of medicinal and poisonous plants: common names, scientific names, eponyms, synonyms, and etymology (5 Volume Set). Boca Raton, FL, USA: CRC Press.

Quiroz D. \& van Andel T., 2015. Evidence of a link between taboos and sacrifices and resource scarcity of ritual plants. J. Ethnobiol. Ethnomed., 11(1), 5.

Réjou-Méchain M. et al., 2011. Detecting large-scale diversity patterns in tropical trees: can we trust commercial forest inventories? For. Ecol. Manage., 261(2), 187-194.

Rondeux J., 1999. La mesure des arbres et des peuplements forestiers. $2^{\mathrm{e}}$ ed. Gembloux, Belgique: Les Presses agronomiques de Gembloux.

ROUGIER, 2001. Plan d'aménagement de l'UFA du HautAbanga. Gabon : FRM.

ROUGIER, 2002. Plan d'aménagement de l'UFA de l'Ogooué Ivindo. Gabon : FRM.

SFID, 2005. Plan d'aménagement (Rougier) de l'UFA 10.054 . Douala, Cameroun : ROUGIER.
Sheil D. et al., 2006. Illumination-size relationships of 109 coexisting tropical forest tree species. J. Ecol., 94(2), 494-507.

Sosef M.S.M., 2006. Check-list des plantes vasculaires $d u$ Gabon. Meise, Belgique : National Botanic Garden of Belgium.

Swaine M.D. \& Hall B.J., 1988. The mosaic theory of forest regeneration and the determination of forest composition in Ghana. J. Trop. Ecol., 4, 253-269.

Vernay M. \& Fouquet D., 2000. Essences tropicales à forte durabilité naturelle. Bois For. Trop., 264, 73-76.

Villiers J.F., 1989. Leguminosae-Mimosoideae. Flore $d u$ Gabon : 31. Paris : Musée National d'Histoire Naturelle.

Vivien J. \& Faure J.J., 1985. Arbres des forêts denses d'Afrique centrale. Paris : ACCT.

Vleminckx J., 2015. The influence of soil factors and anthropogenic disturbances on tree species assemblages in Central African forest. Phd thesis: Université Libre de Bruxelles (Belgique).

White F., 1983. The vegetation of Africa, a descriptive memoir to accompany the UNESCO/AETFAT/UNSO vegetation map of Africa (3 Plates, Northwestern Africa, Northeastern Africa, and Southern Africa, 1: 5,000,000). Paris: United Nations Educational, Scientific and Cultural Organization.

White L. \& Abernethy K., 1996. Guide de la végétation de la Réserve de la Lopé. Libreville : ECOFAC Gabon.

Wieringa J.J., 1999. Monopetalanthus exit.: a systematic study of Aphanocalyx, Bikinia, Icuria, Michelsonia and Tetraberlinia (Leguminosae, Casalpinioideae). Phd thesis: Wageningen Agricultural University (The Netherlands).

WIJMA S.A., 2011. Plan d'aménagement durable UFA 09021. Douala : FORM Ecology Consultants SARL.

Wilks C. \& Issembé Y., 2000. Guide pratique d'identification: les arbres de la Guinée équatoriale. Région continentale. Bata, Guinée équatoriale: Projet CUREF.

(96 réf.) 